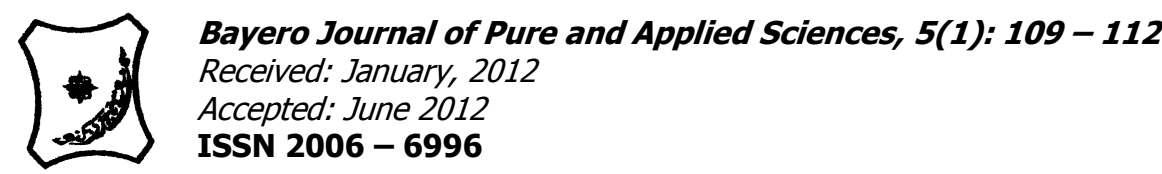

\title{
DETECTION OF EXTENDED-SPECTRUM $\beta$-LACTAMASES AMONG GRAM NEGATIVE ISOLATES FROM GOMBE SPECIALIST HOSPITAL USING DISC REPLACEMENT METHOD
}

\author{
${ }^{1}$ Garba, L. and ${ }^{2} \mathrm{M}$. Yusha'u \\ ${ }^{1}$ Department of Biological Sciences, Gombe State University, Gombe State, Nigeria \\ ${ }^{2}$ Department of Microbiology, Bayero University, Kano, Nigeria
}

\section{ABSTRACT}

A total of 500 clinical bacterial isolates from various sources including stool, urine, sputum and swabs obtained from Gombe State Specialist Hospital between July, 2011 and January, 2012 were used in this study. Gram's stain reaction of the isolates separated them into Gram-positives (200) and Gram-negatives (300). Biochemical tests confirmed the identity of the Gram-negative isolates to be members of the enterobactericeae, which included Klebsiella pneumoniae (60), Escherichia coli (98), Providencia Spp. (32), Morganella moganii (32), Shigella Spp. (14), Citrobacter freundii (14), Serratia marcescens (10), Salmonella paratyphi A (10), Yersinia enterocolitica (8), Proteus vulgaris (4), Salmonella typhi (2) and Pseudomonas aeruginosa (16). Of the 300 Gram-negative

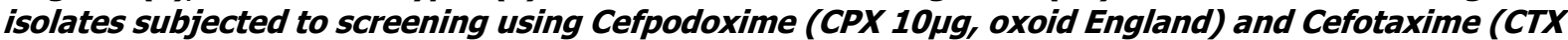
$30 \mu g$, Oxoid England) for ESBL-production based on Clinical Laboratory Standard Institute (CLSI) breakpoint, 250 (83.33\%) were found to be positive which included $K$. pneumoniae (40), E. coli (92), Providencia Spp. (30), M. morganii (20), P. aeruginosa (14), Shigella Spp. (14), C. freundii (12), S. marcescens (6), and $Y$. enterocolitica (6), S. paratyphi A (10), P. vulgaris (4) and S. typhi (2). However, one hundred and sixty four, 164 (65.6\%) were confirmed ESßL-producers based on

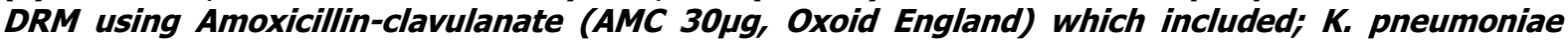
32(19.50\%), E. coli 52(31.71\%), Providencia Spp 20(12.20\%), M. morganii 16(9.76\%), P. aeruginosa 8(4.88\%), Shigella Spp. 12(7.32\%), C. freundii 6(3.66\%), S. marcescens $4(2.44 \%), S$. paratyphi A 8(4.88\%), Y. enterocolitica 6(3.66\%), P. vulgaris $(0.0 \%)$, and S. typhi $(0.0 \%)$. Keywords: Detection, ESBLs, Clinical isolates, Disc Replacement Method, Gombe

\section{INTRODUCTION}

Betalactam antimicrobial agents are the most common drugs for the treatment of bacterial infections and account for over $50 \%$ of global antibiotic usage (Kotra et al., 2002). Beta lactamase production by Gram negatives is the important single mechanism of resistant to beta lactam antimicrobial agents. The beta lactamases are enzymes that hydrolyzed the beta lactam ring of beta lactam antibiotic, thereby rendering them inactive.

Extended- spectrum $\beta$-lactamases (ES $\beta$ Ls) are enzymes that mediate resistance to extendedspectrum (third-generation) Cephalosporins such as Ceftazidime, Cefotaxime, and Ceftriaxone as well as Monobactams sush as Aztreonam (NCCLS, 1999). Organisms that produce ES $\beta$ Ls remain important factor to consider as responsible for therapy failure with Cephalosporins and have serious consequences for infection control (Paterson and Bonomo, 2005).

The extended spectrum $\beta$-lactams became widely used in the treatment of serious infections due to Gram- negative bacteria in 1980's (Bradford, 2001). Resistance to these newer $\beta$-lactams emerge quickly and the first report of plasmid-encoded $\beta$-lactamases capable of hydrolyzing the extended- spectrum cephalosporins was published in 1983 (Bradford, 2001; Samaha-kfoury and Araj, 2003).

ESßLS occur predominantly in the family Enterobacteriaceae leading to outbreaks of nosocomial infections in intensive care units, burn, oncology and neonatal units (Kohler et al., 1999).

A study was conducted to detect ES $\beta$ Ls and determine the ESBL type in clinical isolates of $E$. coli from Granada, Spain. A total of 62 isolates were screened using the Vitek 2 system, Disc diffusion method epsilon test. Fourteen isolates were randomly selected and subjected to genetic analysis in order to detect the ESBL type. Out of the 62 isolates with ESBL detected by Vitek 2 system, 61 (98.4\%) were confirmed to contain ES $\beta$ Ls by disc diffusion and Epsilon test. CTX-M-9 ES $\beta$ L was typed in E. coli (Solorzano et al., 2006).

A research was conducted at Murtala Mohammed Specialist Hospital, Kano, Nigeria to determine the prevalence of ES $\beta$ LS among members of Enterobacteriaceae. Of the 114 Enterobacterial isolates subjected to ESßL detection using CLSI breakpoint, $76(66.7 \%)$ were found to be positive which included Citrobacter spp.(4), Enterobacter spp. (3), Escherichia coli (28), Klebsiella spp. (18), Morganella morganii (7), Proteus spp.(13), Salmonella spp.(1), Serratia spp.(1), Shigella spp. (2) and Yersinia spp.(1). However, 47(41.2\%) were confirmed to be ESBL producers based on DRM which included; Citrobacter spp. (1), E. coli (20), Klebsiella spp. (12), M. morganii (4), Proteus spp.(8), Salmonella spp. (1) and Shigella spp. (1) (Yusha'u et al., 2010). 


\section{MATERIALS AND METHODS}

Bacterial isolates

A total of 500 bacterial isolates obtained from Gombe State specialist Hospital were used for this study.

\section{Reaction of the isolates to Gram's staining}

The bacterial isolates were subjected to Gram's stain reactions and separated into Gram positives and Gram negatives using the standard procedure described by Cheesebrough, (2000).

\section{Identification of the Gram negative isolates based on biochemical tests}

The Gram negative isolates were subjected to biochemical tests which indentified them to be members of the family Enterobacteriaceae and Pseudomonas aeruginosa using the standard procedure including Urease test, citrate utilization test, indole test and Kligler Iron Agar test as described by Cheesebrough, (2000).

\section{Inoculum standardization}

This was performed according to suggestion made by NCCLS, (1999). The Gram negative isolates were sub cultured onto prepared Brain Heart Infusion (BHI) agar. Following incubation at $37^{\circ} \mathrm{C}$ for $24 \mathrm{hrs}$, a few loopful of the isolates were dispensed in a sterile normal saline to match the 0.5 McFarland standards for sensitivity.

Clinical Laboratory Standard Institute (CLSI) breakpoint test for ES $\beta L$ s screening

All the Gram negative isolates were subjected to screening for ESBLS production using Cefpodoxime

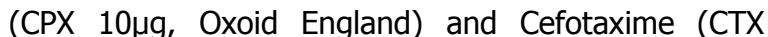

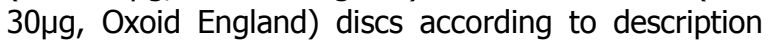
by NCCLS, (1999).

\section{Disc Replacement Method (DRM)}

Suspected ES $\beta$ Ls producers based on CLSI were subjected to confirmatory test using DRM described by Casal and Pringler, (1990). Two Amoxicillinclavulanate (Augmentin) discs (AMC 30 $\mathrm{\mu g}$, Oxoid England) were placed on prepared Muller-Hinton agar plates inoculated with the Gram negative isolates. After an hour at room temperature, the discs were removed and replaced at the spot of each with Cefotaxime (CTX 30g) and Cefpodoxime (CPX 10g), incubated at $37^{\circ} \mathrm{C}$ for $24 \mathrm{hrs}$ before they were read for the evidence of ES $\beta L S$ production (increase in zone diameter of $\geq 5 \mathrm{~mm}$ from that recorded during screening of confirmed ES $\beta$ L production).

\section{RESULTS}

Biochemical identification of the clinical isolates revealed higher number of Gram-negatives than Gram-positive organisms as shown in Table 1.

Screening of the isolates for the presence of ESBL indicated that the enzymes occur at higher rate among $E$. coli $(36.8 \%)$ than the other isolates while the least occurrence rate was observed among Salmonella typhi $(0.8 \%)$ (Table 2$)$.

On subjecting the isolates positive for screening test to confirmation using disc replacement method, the highest occurrence rate was observed among $E$. coli $(31.7 \%)$ than the other isolates while the least occurrence rate was observed among Salmonella typhi and Proteus vulgaris with $0 \%$ each (Table 3 ).

Table 1: Gram's staining reactions of the bacterial isolates

\begin{tabular}{lcc}
\hline Gram's reaction & Number observed & \% occurrence \\
\hline Positive & 200 & 40 \\
Negative & 300 & 60 \\
\hline Total & $\mathbf{5 0 0}$ & $\mathbf{1 0 0}$ \\
\hline
\end{tabular}

Table 2: Percentage occurrence of ES $\beta$ Ls producers among the isolates based on CLSI

\begin{tabular}{lccc}
\hline Isolate & Number screened & Number positive & \% occurrence \\
\hline Klebsiella pneumoniae & 60 & 40 & 16 \\
Escherichia coli & 98 & 92 & 36.8 \\
Providencia spp & 32 & 30 & 12 \\
Morganella morganii & 32 & 20 & 8 \\
Psuedomonas aeruginosa & 16 & 14 & 5.6 \\
Shigella spp & 14 & 14 & 5.6 \\
Citrobacter freundii & 14 & 12 & 4.8 \\
Serratia marcescens & 10 & 6 & 2.4 \\
Salmonella paratyphi $A$ & 10 & 10 & 4 \\
Yersinia enterocolitica & 8 & 6 & 2.4 \\
Proteus vulgaris & 4 & 4 & 1.6 \\
Salmonella typhi & 2 & 2 & 0.8 \\
\hline Total & $\mathbf{3 0 0}$ & $\mathbf{2 5 0}$ & $\mathbf{8 3 . 3 3}$ \\
\hline
\end{tabular}


Table 3: Confirmed percentage occurrence of ES $\beta$ Ls producers among the isolates based on Disc Replacement Method (DRM)

\begin{tabular}{lccc}
\hline Isolates & Number screened & Number positive & \% occurrence \\
\hline K. pneumoniae & 40 & 32 & 19.50 \\
E. coli & 92 & 52 & 31.71 \\
Providencia spp & 30 & 20 & 12.20 \\
M. morganii & 20 & 16 & 9.76 \\
P. aeruginosa & 14 & 8 & 4.88 \\
Shigella spp & 14 & 12 & 7.32 \\
C. freundii & 12 & 6 & 3.66 \\
S. marcescens & 6 & 4 & 2.44 \\
S. paratyphi $A$ & 10 & 8 & 4.88 \\
Y. enterocolitica & 6 & 6 & 3.66 \\
P. vulgaris & 4 & 0 & 0 \\
S. typhi & 2 & 0 & 0 \\
\hline Total & $\mathbf{2 5 0}$ & $\mathbf{1 6 4}$ & $\mathbf{6 5 . 6}$ \\
\hline
\end{tabular}

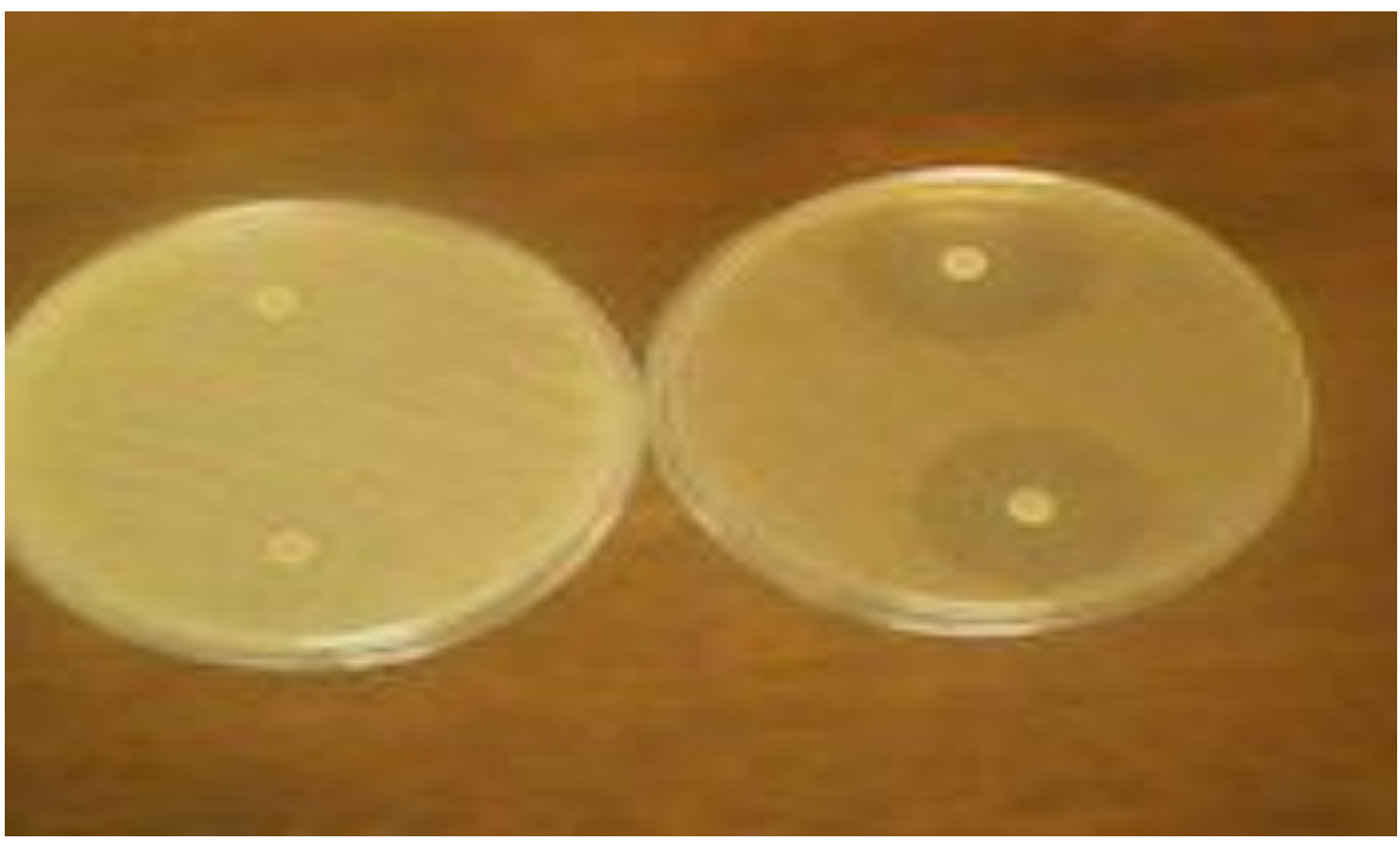

Plate 1: Positive CLSI breakpoint screening test (Left) and confirmed ES $\beta$ L producer based on DRM (Right).

\section{DISCUSSION}

Of the 500 clinical bacterial isolates subjected to Gram's staining reactions, Gram negative isolates were the most predominant in occurrence with 300 $(60 \%)$ over the Gram positive isolates with only 200 $(40 \%)$. The high occurrence of Gram negative observed may be related to improper hygiene from the side of the patient which can result in possible outbreak of infection.

On subjecting the Gram negative isolates to screening test for occurrence of ES $\beta$ Ls based on CLSI breakpoint, $250(83.33 \%)$ were found to be positive which included; K. pneumoniae (40), E. coli (92), Providencia spp (30), M. morganii (20), P. aeruginosa (14), Shigella spp (14), C. freundii (12), S. marcescens (6), S. paratyphi $A(10), Y$. enterocolitica (6), $P$. vulgaris (4) and $S$. typhi (2). However, of the 250 positives in the screening test, one hundred and sixty four, $164(65.6 \%)$ were confirmed to be ESßLproducers based on DRM (Table 3 ). The high occurrence of ESBLs producers among the clinical isolates indicates possible treatment failures using broad spectrum antibiotics directed against common resistant organisms.

Among the ES $\beta$ Ls producers detected, E. coli recorded the highest occurrence rate of 52 (31.71\%) followed by $K$. pneumoniae with $32(19.50 \%)$ which conforms to the findings of Yusha'u et al., (2010) (E. coli, 20 and $K$. pneumoniae, 12). Others were Providencia spp (20), M. morganii (16), P. aeruginosa (8), Shigella spp (12), C. freundii (6), S. marcescens (4), S. paratyphi $A$ (8), and $Y$. enterocolitica (6). However, there was no evidence of ES $\beta$ L production observed in the clinical isolates of $P$. vulgaris and $S$. typhi based on the confirmatory test used (DRM). This agrees with the findings of Akujobi et al., (2010) in a study conducted in the sourth-Eastern Nigeria where they detected no evidence of ES $\beta$ LS among the 6 isolates of Proteus spp. tested. 


\section{CONCLUSION}

From this research, it can be concluded that the percentage occurrence of the ESBLs detected at the study site was high with the calculated value (187.07) being greater than the table value (11.07) at $P=0.05$ using chi-square statistical analysis which calls for an urgent attention as there may be possibility of treatment failure with cephalosporins.

\section{RECOMMENDATIONS}

(a) Efforts should be made to determine the $\mathrm{ES} \beta \mathrm{L}$ genotypes using molecular techniques.

\section{REFERENCES}

Akujobi, C., N., and Ewunu, C., P. (2010): detection of extended spectrum beta-lactamases in gramnegative bacilli from clinical specimens in a teaching hospital in south eastern Nigeria. Niger med J. 51:141-6.

Bradford, P. A. (2001): Extended-spectrum $\beta$ lactamases in the $21^{\text {st }}$ century, Characteristics, epidemiology and detection of this important resistance threat. Clin microbial rev 14: 933-951.

Casal, I., and Pringler, J. (1990): Mediterranian congress of Chemothepy, Barcelona, spain, 20 to25 may, 1990.

Cheesebrough, M. (2000): District laboratory practice in tropical countries, part 2, Cambridge university press: $38-70$

Kohler, J. Dorso, K., L., and Young, K. (1999): In vitro activities of the potent broad spectrum Carbapenem MK0826(L749,345) against broad-spectrum beta-lactamase and extended spectrum beta lactamase-producing Klebsiella pneumoniae and E. coli clinical isolates. Antimicrob. Agents chemothe 43:1170-1176.

Kotra, L., P., Samania, J., and Mobashery, S. (2002): $\beta$-lactamases and resistance to $\beta$-Lactam antibiotics. In Lewis, K., Salyers, A. A. Tabar, (b) Control measures such as proper handling and disinfection of equipments as well as detection of ES $\beta$ LS producing organisms among the patients and isolation of ESßLS colonized patients should be improved by the Health care settings.

(c) Awareness campaigns on improved hygienic practices should be diversified by both governmental and Non-governmental Organizations so as to reduce the rate of infections and spread of ES $\beta$ Ls among both enterobacterial and other bacterial pathogens.

H. W. and wax, R. G.Eds. Bacterial resistances to antimicrobials. Marcel Decker, New York.123-60.

National Committee for clinical Laboratory Standard (1999): Performance standard for antimicrobial susceptibility testing. National community for clinical laboratory Approved standard M 106- 59.

Paterson, D., L., and Bonomo, R. A. (2005): Extendedspectrum $\beta$-lactamases:aclinical Update. Clin microbial rev. 18:657-686.

Samaha-kfoury, J., N. and Araj, G., F. (2003): Recent development in $\beta$-lactamases and Extended spectrum b-lactamases. $\mathrm{Br}$ med J 327: 12091213

Solorzano, A., Gutierez, J., F., Soto, M., J., Piedrola, G. (2006): A preliminary studyon the presence of extended spectrum beta-lactamases (ESßLS) in clinical isolate of $E$. coli in Granda, Spain. CAB Abstract.

Yusha'u, M. Aliyu, H. M., Kumurya, A. S. and Suleiman, K. (2010): Prevalence of Extended spectrum $\beta$-lactamases (ESBLs) among Enterobacteriacea in Murtala Mohammed specialist Hospital, Kano, Nigeria. Bayero journal of pure and Applied Sciences, 3(1): 169-172. 